\title{
Inseminação artificial em tempo fixo e diagnóstico precoce de gestação em vacas leiteiras mestiças ${ }^{1}$
}

\author{
Cláudio França Barbosa², José Octavio Jacomini², Elmo Gomes Diniz ${ }^{2}$, Ricarda Maria dos \\ Santos $^{2}$, Marcelo Tavares ${ }^{3}$
}

\author{
1 Projeto financiado pela FAPEMIG - APQ - 1601/07. \\ 2 FAMEVIUFU, Uberlândia, MG, Brasil. \\ ${ }^{3}$ FAMAT/UFU, Uberlândia, MG, Brasil.
}

RESUMO - Avaliou-se, durante um ano, o desempenho reprodutivo de 94 vacas leiteiras mestiças Bos taurus $\times$ Bos indicus submetidas a um programa de reprodução assistida. Um protocolo de inseminação artificial em tempo fixo (IATF) foi executado por meio de dispositivo intravaginal contendo progesterona e das injeções de prostaglandina F2 $\alpha$ e de cipionato de estradiol. Por meio de ultrassonografia, entre 7 e 14 dias após as inseminações ou montas controladas, realizou-se a detecção de corpo lúteo nos ovários a fim de determinar a taxa de ovulação e, no $28^{0}$ dia, fez-se o diagnóstico de gestação para cálculo da taxa de concepção. Respeitou-se um período mínimo de 34 dias após o parto antes do tratamento. Não houve influência do escore de condição corporal e da presença de corpo lúteo no início do protocolo, nem da reutilização do dispositivo intravaginal e da monta controlada ou inseminação artificial, sobre as taxas de ovulação, concepção e concepção das vacas ovuladas. As taxas de concepção e de concepção das vacas ovuladas foram afetadas negativamente pelo elevado número de dias pós-parto (DPP), ou dias em lactação e pela época quente do ano, primavera/verão. A resposta ao protocolo de inseminação artificial em tempo fixo baseado no uso de progesterona, PGF2 $\alpha$ e cipionato de estradiol é prejudicada pelo aumento dos dias em lactação e pela época quente do ano. A condição corporal não afeta a resposta ao protocolo de inseminação artificial, desde que as vacas tratadas apresentem escore acima de 2,25 pontos.

Palavras-chave: concepção, eficiência reprodutiva, ovulação, protocolo de sincronização

\section{Timed artificial insemination and early pregnancy diagnosis in crossbred dairy cows}

\begin{abstract}
It was evaluated, during a period of one year, the reproductive performance of 94 Bos taurus $\times$ Bos indicus crossbred dairy cows submitted to an assisted reproduction program. A timed artificial insemination (TAI) protocol was carried out by using an intra-vaginal progesterone device containing progesterone and through injections with Prostaglandin F2 $\alpha$ and estradiol cypionate. By using ultrasound, from 7 to 14 days after inseminations or controlled mattings, detection of corpus luteum in the ovaries was done to determine ovulation rate and, on the $28^{\text {th }}$ day, diagnosis of pregnancy was carried out for calculation of conception rate. Treatment only started after a 34-day post-partum minimal period. Body condition score, presence of corpus luteum in the beginning of the protocol, reutilization of intra-vaginal device and controlled mating or artificial insemination did not influence rates of ovulation, conception and conception of ovulated cows. Rates of conception and rates of conception of ovulated cows were negatively affected by the higher number of days post partum (DPP), or days in milk and by the warmer seasons of the year, spring/summer. Response to protocol of timed artificial insemination based on the use of progesterone, PGF2 $\alpha$ and estradiol cypionate is negatively affected by lactation days and by the warm season of the year. Body condition does not affect the response to protocol of artificial insemination provided that treated cows show body condition scores higher than 2.5 points.
\end{abstract}

Key Words: conception, ovulation, protocol of synchronization, reproductive efficiency

\section{Introdução}

A eficiência reprodutiva é um dos fatores que mais contribui para melhorar o desempenho e a lucratividade dos rebanhos leiteiros (Grohn \& Rajala-Schultz, 2000).
Enfermidades puerperais e metabólicas (Stevenson \& Call, 1988), bem como falhas na detecção de estro (Esslemont, 1993), reduzem o desempenho reprodutivo e, indiretamente, a produção de leite por dia de vida útil da vaca (Britt, 1985). A reduzida taxa de prenhez e maior intervalo de partos 
diminui a produção de bezerros, aumenta as despesas de manutenção das vacas secas e as taxas de descarte.

Vacas em lactação submetidas à temperatura ambiente e umidade relativa do ar elevadas apresentam redução da manifestação de estro, das taxas de ovulação e prenhez (Hansen \& Arechiga, 1999; Lopez-Gatius et al., 2005). Pursley et al. (1997) relataram um menor impacto da baixa eficiência de detecção de estro em vacas lactantes obtido com uso de protocolos de sincronização da ovulação e inseminação artificial em tempo fixo (IATF). As mestiças têm atraso no retorno à ciclicidade (Ruas et al., 2002), portanto são recomendados protocolos de inseminação artificial em tempo fixo que utilizam implantes de $\mathrm{P} 4$ ou progestágenos. Com isso, aumenta-se a taxa de sincronização e, também se previne a ocorrência de ciclos curtos (Perry et al., 2004; Baruselli et al., 2006). Melhor taxa de prenhez, em vacas tratadas com cipionato de estradiol para induzir a ovulação em um protocolo de inseminação artificial em tempo fixo, é esperada quando mais vacas manifestam estro, aumentando assim a taxa de ovulação (Galvão et al., 2004).

O exame ultrassonográfico, entre os dias sete e quatorze após a inseminação, ao identificar vacas que falharam em ovular e não terão chances de se tornarem gestantes, permite resincronizá-las e reinseminá-las o mais cedo possível. O diagnóstico da gestação por ultrassonografia 28 dias após a inseminação objetiva identificar as vacas prenhes precocemente e propiciar a reinseminação das vazias, reduzir o intervalo entre as inseminações e, desse modo, o intervalo do parto à concepção (Vasconcelos \& Garcia, 2006).

A hipótese deste trabalho foi de que o desempenho reprodutivo de vacas leiteiras mestiças Bos taurus $\times$ Bos indicus sofreria influência de número de dias pós-parto (dias em lactação), presença de corpo lúteo, escore de condição corporal, época do ano, número de uso do dispositivo intravaginal com progesterona e do tipo de cobertura (monta controlada ou inseminação artificial). Objetivou-se avaliar o efeito destas variáveis sobre as taxas de ovulação, de concepção e de concepção das vacas ovuladas num programa de reprodução assistida.

\section{Material e Métodos}

A pesquisa foi realizada na Fazenda Experimental do Glória, pertencente à Universidade Federal de Uberlândia, situada na Latitude 1856'54.39" Sul, Longitude 48¹2'47.40" Oeste, 650 a $665 \mathrm{~m}$ acima do nível do mar. As vacas foram mantidas a pasto no período das águas (metade final da primavera e verão) em piquetes de Brachiaria brizantha próximos à sala de ordenha e receberam silagem de milho/ sorgo na seca (outono, inverno e metade inicial da primavera). A suplementação concentrada foi administrada diariamente durante as ordenhas, de acordo com a produção individual de cada vaca. O calendário zoossanitário foi seguido regularmente para todo o rebanho da fazenda obedecendo à legislação sanitária estadual para bovinos.

Foram utilizadas 94 fêmeas lactantes mestiças Bos taurus $\times$ Bos indicus, de maio de 2007 a maio de 2008 . Executou-se o protocolo descrito por Cardoso et al. (2006) 155 vezes (Figura 1), em 16 momentos diferentes. Uma vaca que possuía corpo lúteo e escore de condição corporal 2,75 na sua avaliação pré-protocolo acima de 90 dias em lactação perdeu o dispositivo intravaginal. As épocas do ano avaliadas corresponderam a dois períodos de seis meses cada, representados pelas estações outono/inverno e primavera/verão.

Antes de iniciar cada protocolo as vacas foram selecionadas, para que fossem sincronizadas somente as consideradas aptas. Qualquer sinal evidente de desordem

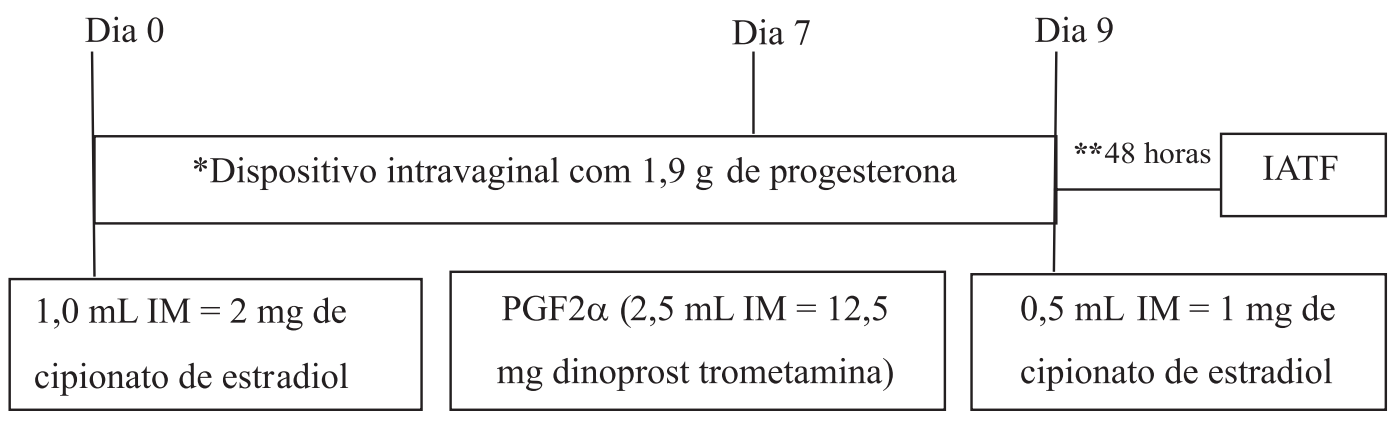

* CIDR - Cada dispositivo intravaginal de P4 (DIP4) foi utilizado por até três vezes.
** Período de deteç̧ão de estro com monta controlada ou inseminação artificial 12 horas após detecção.

Figura 1 - Protocolo de Cardoso et al. (2006) para inseminação artificial em tempo fixo (IATF) ou monta controlada (MC) em vacas lactantes. 
de saúde as excluía do estudo. Os critérios para utilização das vacas foram mais de 34 dias pós-parto em lactação, escore de condição corporal e $\geq 2,25$ e $\leq 3,5$ (escala 1-5, sendo 1 muito magra e 5 muito gorda, segundo Ferguson et al., 1994), útero sem sinais de infecções ao exame clínico e ultrassonográfico (aparelho de ultrassom, transdutor retal linear de 7,5-MHz). Essas avaliações foram realizadas a cada 21 dias.

No dia 0, após a avaliação pré-protocolo, introduziu-se um dispositivo intravaginal de progesterona (DIP4) com 1,9 g (CIDR ${ }^{\circledR}$, Controlled Intravaginal Drug Releasing) e aplicou-se $1 \mathrm{~mL}$ (2 mg) de cipionato de estradiol $\left(\mathrm{ECP}^{\circledR}\right)$ via intramuscular, nas vacas consideradas aptas, para sincronização da onda de desenvolvimento folicular. No dia 7, a luteólise foi induzida com injeção de $2,5 \mathrm{~mL}$ de prostaglandina (Lutalyse ${ }^{\circledR}$, PGF2 $\alpha-12,5$ mg de dinoprost trometamina), via intramuscular. No dia 9, para induzir a ovulação, foi injetado $0,5 \mathrm{~mL}$ (1 mg) de cipionato de estradiol, via intramuscular e retirou-se DIP4, o qual foi submetido à lavagem e secagem para futuro reaproveitamento.

Após a retirada do DIP4 iniciou-se a observação de estro. As vacas detectadas em estro foram imediatamente colocadas com um dos dez touros disponíveis da fazenda ou inseminadas 12 horas depois. Aquelas não detectadas foram inseminadas em tempo fixo (48 horas após a retirada do DIP4). Durante o ano de experimento, doses de 21 touros diferentes foram disponibilizadas para inseminação artificial. O critério que definiu previamente o touro ou a dose sêmen usada na cobrição de cada fêmea foi a genealogia a fim de evitar o aumento do grau de sangue de uma mesma raça e os acasalamentos consanguíneos. Não se relacionaram as variáveis independentes (dias pós-parto em lactação, presença de corpo lúteo, escore de condição corporal, época do ano, uso de DIP4 e tipo de cobertura [monta controlada ou inseminação artificial]) com a detecção ou não de estro após o protocolo e nem com o touro ou sêmen utilizado.

Foram realizadas avaliações pós-protocolo das vacas inseminadas por meio dos exames ultrassonográficos com a finalidade de se identificar a presença de corpo lúteo entre sete e 14 dias após a inseminação para determinar a taxa de ovulação e possibilitar resincronização das que não ovularam, mas foram consideradas aptas. Fez-se o diagnóstico de gestação nas vacas com mínimo de 28 dias de inseminadas para determinar a taxa de concepção e as que permaneceram vazias foram novamente sincronizadas e resubmetidas á monta controlada ou inseminação artificial.

A resposta ao protocolo de sincronização foi calculada por meio das taxas de ovulação, de concepção e de concepção das vacas ovuladas. Realizou-se o teste nãoparamétrico da binomial para duas proporções na comparação das porcentagens. Foi usada uma significância de 5\% para determinar a ocorrência das diferenças estatísticas do efeito das variáveis independentes sobre as taxas de ovulação, concepção e concepção das ovuladas.

\section{Resultados e Discussão}

A taxa de ovulação após o protocolo foi de $90,26 \%$ (139/154) e não sofreu influência de nenhuma das variáveis analisadas (Tabelas 1 a 6). Esse valor foi numericamente acima do encontrado por Vasconcelos et al. (1999) e Santos \& Vasconcelos (2008), que obtiveram 87,0 e 80,9\% de sincronização de ovulação, respectivamente, quando trabalharam com vacas lactantes da raça Holandesa tratadas com o protocolo Ovsynch. Estes resultados foram obtidos, provavelmente, devido a melhor resposta das vacas mestiças tratadas com progesterona, PGF2 $\alpha$ e cipionato de estradiol.

Não foi detectado efeito de número de dias pós-parto ou de dias em lactação na taxa de ovulação (Tabela 1), diferindo dos resultados encontrados por Demétrio et al. (2007), que verificaram que com o aumento do número de dias pós-parto, houve redução da probabilidade de ovulação em vacas Holandesas de alta produção, o que não foi observado neste estudo, provavelmente devido ao uso de vacas mestiças de menor produção. As vacas com dias pós-parto ou dias em lactação menor que 90 tiveram maior taxa de concepção do que as com mais de 90 dias $(\mathrm{P}<0,05$; Tabela 1), diferindo dos resultados de Tenhagen et al. (2003), que, ao usarem o protocolo Ovsynch sem DIP4, relataram que vacas inseminadas em tempo fixo, com maior número de dias pós-parto ou dias em lactação, tiveram maior taxa de concepção ao primeiro serviço, quando comparadas a outras

Tabela 1 - Dias pós-parto ou dias em lactação e desempenho reprodutivo de vacas leiteiras mestiças lactantes tratadas com progesterona, PGF2 $\alpha$ e cipionato de estradiol

\begin{tabular}{|c|c|c|c|c|c|}
\hline $\begin{array}{l}\text { Dias pós-parto/ } \\
\text { dias em lactação }\end{array}$ & $\begin{array}{c}\text { № de } \\
\text { animais* }\end{array}$ & $\begin{array}{c}\text { Taxa de } \\
\text { ovulação (\%) }\end{array}$ & $\begin{array}{c}\text { Taxa de } \\
\text { concepção (\%) }\end{array}$ & $\begin{array}{l}\text { № de } \\
\text { animais }\end{array}$ & $\begin{array}{c}\text { Taxa de concepção das } \\
\text { vacas ovuladas (\%) }\end{array}$ \\
\hline $34-90$ & 82 & $90,24 a$ & $43,90 a$ & 74 & $48,64 a$ \\
\hline$>90$ & 71 & $88,73 a$ & $23,94 b$ & 63 & $26,98 b$ \\
\hline
\end{tabular}

Letras distintas na mesma coluna diferem $(\mathrm{P}<0,05)$. * Duas vacas não apresentavam registro de data do último parto quando foi determinado o número de dias pós-parto. 
inseminadas com três semanas a menos, independentemente do nível de produção de leite. Essas diferenças de efeito dos dias pós-parto, ou dias em lactação na resposta aos protocolos, provavelmente ocorreram devido às diferenças do material e métodos, já que, neste estudo, foram utilizados dispositivos intravaginais de progesterona, o que favoreceu a concepção das vacas com dias pós-parto ou dias em lactação menor que 90 .

As taxas de concepção e de concepção das vacas ovuladas foram influenciadas pelos dias pós-parto ou dias em lactação ( $\mathrm{P}<0,05$; Tabela 1$)$. Chebel et al. (2004) e Demetrio et al. (2007) também observaram taxas de prenhez menores em vacas com dias pós-parto ou dias em lactação mais avançado. Isso ocorreu porque as vacas que não conceberam até 90 dias após o parto, provavelmente apresentaram algum tipo de problema não diagnosticado.

Em vacas mestiças há maior ocorrência de três ondas de crescimento folicular em cada ciclo estral, o que diminui a resposta à primeira e à segunda aplicação de GnRH e, conseqüentemente, reduz a taxa de ovulação após o protocolo Ovsynch (Vasconcelos et al., 1999). Neste estudo como foi utilizado cipionato de estradiol no início do protocolo, houve melhor sincronismo do início da nova onda de crescimento, o que resultou em maior taxa de ovulação ao final do protocolo.
A presença ou ausência de corpo lúteo observada ao iniciar o protocolo usado nesta pesquisa, não afetou os resultados de taxas de ovulação, de concepção ou de concepção das vacas ovuladas (Tabela 2), o que confirma os resultados de Martel (2008) e Souza et al. (2009) que não encontraram diferença na taxa de concepção das vacas com presença ou ausência de corpo lúteo, no momento da inserção da P4 exógena. A progesterona presente no dispositivo provavelmente foi suficiente tanto para promover a produção de um ovócito saudável, pois já foi demonstrado a correlação entre a concentração sérica de progesterona antes da inseminação artificial e a subsequente taxa de concepção (Fonseca et al., 1983; Folman et al.,1990), como para inibir a ocorrência de ciclo curto nas vacas sem corpo lúteo ao início do protocolo, que estavam em anestro (Fortune, 1994; Rivera et al., 1998).

Não foi detectado efeito do escore de condição corporal sobre a taxa de ovulação ou sobre as taxas de concepção ou de concepção das vacas ovuladas (Tabela 3; $\mathrm{P}>0,05$ ), o que difere dos resultados obtidos por Santos et al. (2009), que observaram que a taxa de concepção foi afetada negativamente pelo baixo escore de condição corporal ao parto e a primeira inseminação artificial. Neste estudo não foi detectado efeito da condição corporal provavelmente porque as vacas com escore menor que 2,0 não foram

Tabela 2 - Presença de corpo lúteo e desempenho reprodutivo de vacas leiteiras mestiças lactantes tratadas com progesterona, PGF2 $\alpha$ e cipionato de estradiol

\begin{tabular}{lccccc}
\hline Ciclicidade & No de & Taxa de & \multicolumn{2}{c}{ Taxa de } & No de \\
animais & ovulação (\%) & Taxa de concepção das & concepção (\%) & vacas ovuladas (\%) \\
\hline Presença de corpo lúteo & 89 & $92,13 \mathrm{a}$ & $32,58 \mathrm{a}$ & 82 \\
Ausência de corpo lúteo & 66 & $86,36 \mathrm{a}$ & $39,39 \mathrm{a}$ & 57 \\
\hline
\end{tabular}

Letras distintas na mesma coluna diferem $(\mathrm{P}<0,05)$.

Tabela 3 - O escore de condição corporal (ECC) e desempenho reprodutivo de vacas leiteiras mestiças lactantes tratadas com progesterona, PGF2 $\alpha$ e cipionato de estradiol

\begin{tabular}{lccccc}
\hline ECC & $\begin{array}{c}\text { № de } \\
\text { animais }\end{array}$ & $\begin{array}{c}\text { Taxa de } \\
\text { ovulação (\%) }\end{array}$ & $\begin{array}{c}\text { Taxa de } \\
\text { concepção (\%) }\end{array}$ & $\begin{array}{c}\text { № de } \\
\text { animais }\end{array}$ & $\begin{array}{c}\text { Taxa de concepção das } \\
\text { vacas ovuladas (\%) }\end{array}$ \\
\hline$\leq 2,50$ & 85 & $89,41 \mathrm{a}$ & $31,76 \mathrm{a}$ & 76 & $35,53 \mathrm{a}$ \\
$\geq 2,75$ & 69 & $91,3 \mathrm{a}$ & $40,58 \mathrm{a}$ & 63 & $44,44 \mathrm{a}$ \\
\hline
\end{tabular}

Letras distintas na mesma coluna diferem $(\mathrm{P}<0,05)$.

Tabela 4 - Estações do ano e o desempenho reprodutivo de vacas leiteiras mestiças lactantes tratadas progesterona, PGF2 $\alpha$ e cipionato de estradiol

\begin{tabular}{|c|c|c|c|c|c|}
\hline Estações do ano & $\begin{array}{l}\text { № de } \\
\text { animais }\end{array}$ & $\begin{array}{c}\text { Taxa de } \\
\text { ovulação (\%) }\end{array}$ & $\begin{array}{c}\text { Taxa de } \\
\text { concepção (\%) }\end{array}$ & $\begin{array}{l}\text { No de } \\
\text { animais }\end{array}$ & $\begin{array}{c}\text { Taxa de concepção das } \\
\text { vacas ovuladas (\%) }\end{array}$ \\
\hline Outono/inverno & 94 & $92,55 a$ & $42,55 a$ & 87 & $45,98 a$ \\
\hline Primavera/verão & 60 & $86,67 a$ & $25,00 b$ & 52 & $28,85 b$ \\
\hline
\end{tabular}

Letras distintas na mesma coluna diferem $(\mathrm{P}<0,05)$. 
tratadas, e a porcentagem de vacas com escore muito alto era muito baixa.

A estação do ano não afetou a taxa de ovulação ( $\mathrm{P}>0,05$; Tabela 4), o que diferiu dos resultados encontrados por Lopez-Gatius et al. (2005), que observaram falhas de ovulação de 3,4\% em estações com temperaturas ambientais até $25^{\circ} \mathrm{C}$ e $12,4 \%$ quando as temperaturas foram superiores a $25^{\circ} \mathrm{C}$. Detectou-se efeito da estação do ano (Tabela 4; $\mathrm{P}<0,05)$ nas taxas de concepção ou de concepção das vacas ovuladas. A concepção foi maior nos meses de outono/ inverno (42,55\%) que nos meses de primavera/verão $(25,0 \%)$, mostrando que vacas mestiças, apesar de mais resistentes ao estresse térmico, também sofrem os efeitos negativos da temperatura e da umidade do ar mais altas.

Não se detectou efeito do número de utilizações do dispositivo intravaginal de progesterona nos índices reprodutivos analisados ( $\mathrm{P}>0,05$; Tabela 5). Observou-se que estes dispositivos puderam ser reutilizados sem prejuízo para os resultados, o que diferiu dos relatos de Dias et al. (2009) ao trabalharem com novilhas Nelore. Estes autores encontraram maior taxa de prenhez após inseminação artificial em tempo fixo em animais tratados com dispositivo intravaginal de terceiro uso. Essa diferença ocorreu possivelmente porque, no presente estudo, foram utilizadas vacas mestiças em lactação e a concentração de progesterona fornecida pelo dispositivo provavelmente não prejudicou o desenvolvimento folicular. Esse resultado confirma que o dispositivo intravaginal com $1,9 \mathrm{~g}$ de progesterona pode ser utilizado por até três vezes sem afetar a taxa de ovulação e de concepção após a inseminação artificial em tempo fixo em vacas leiteiras.

Tabela 5 - Reutilização de dispositivo intravaginal de progesterona e desempenho reprodutivo de vacas leiteiras mestiças lactantes tratadas com PGF2 $\alpha$ e cipionato de estradiol

\begin{tabular}{|c|c|c|c|c|c|}
\hline Uso do DIP4 & $\begin{array}{l}\text { № de } \\
\text { animais }\end{array}$ & $\begin{array}{c}\text { Taxa de } \\
\text { ovulação (\%) }\end{array}$ & $\begin{array}{c}\text { Taxa de } \\
\text { concepção (\%) }\end{array}$ & $\begin{array}{l}\text { № de } \\
\text { animais }\end{array}$ & $\begin{array}{c}\text { Taxa de concepção das } \\
\text { vacas ovuladas (\%) }\end{array}$ \\
\hline $1^{\underline{0}}$ (0-9 dias) & 56 & $83,92 \mathrm{a}$ & $33,92 \mathrm{a}$ & 47 & $40,42 \mathrm{a}$ \\
\hline $2^{\circ}$ (10-18 dias) & 54 & $88,88 a$ & $36,36 a$ & 48 & $41,66 \mathrm{a}$ \\
\hline
\end{tabular}

Letras distintas na mesma coluna diferem $(\mathrm{P}<0,05)$. DIP4 - Dispositivo intravaginal de progesterona (CIDR ${ }^{\circledR}$ - Pfizer Saúde Animal).

Tabela 6 - Taxa de concepção de vacas leiteiras mestiças lactantes submetidas a protocolo de inseminação artificial em tempo fixo com progesterona, PGF2 $\alpha$ e cipionato de estradiol

\begin{tabular}{lccccc}
\hline Tipo de cobertura & $\begin{array}{c}\text { № de } \\
\text { animais }\end{array}$ & $\begin{array}{c}\text { Taxa de } \\
\text { ovulação (\%) }\end{array}$ & $\begin{array}{c}\text { Taxa de } \\
\text { concepção (\%) }\end{array}$ & $\begin{array}{c}\text { № de } \\
\text { animais }\end{array}$ & $\begin{array}{c}\text { Taxa de concepção das } \\
\text { vacas ovuladas (\%) }\end{array}$ \\
\hline Monta controlada & 58 & 86,21 & $34,48 \mathrm{a}$ & 50 & $40,00 \mathrm{a}$ \\
Inseminação artificial & 95 & 92,63 & $35,78 \mathrm{a}$ & 88 & $38,64 \mathrm{a}$ \\
\hline
\end{tabular}

Letras distintas na mesma coluna diferem $(\mathrm{P}<0,05)$.

O tipo de cobertura não afetou as taxas de ovulação, de concepção ou de concepção das vacas ovuladas ( $\mathrm{P}>0,05$; Tabela 6). Demonstrou-se que esse protocolo pode ser utilizado com monta natural, desde que seja respeitado o número de montas diárias de cada touro. No presente experimento, foram utilizados dez touros, que faziam apenas uma cobertura por dia.

Neves et al. (2010) atribuíram às pesquisas dos mecanismos envolvidos no controle da fisiologia reprodutiva, em níveis endócrino, celular e molecular, maior evolução das biotécnicas reprodutivas na primeira década do século XXI. Este experimento comprova a afirmação destes autores. Destaca-se a necessidade fundamental de mais incentivo a pesquisas considerando os principais fatores de manejo reprodutivo, nutricional e sanitário, que afetam a produtividade da pecuária leiteira, para que IATF, a transferência de embriões (TE), produção in vitro de embriões (PIVE), clonagem e transgênese sejam aprimoradas e continuem auxiliando o desenvolvimento da pecuária nacional.

\section{Conclusões}

A concepção de vacas leiteiras mestiças tratadas com progesterona, PGF2 $\alpha$ e cipionato de estradiol é afetada negativamente pelo número de dias em lactação e pela época quente do ano (primavera/verão). A condição corporal não afeta a resposta ao protocolo de inseminação artificial em tempo fixo desde que as vacas tratadas apresentem escore superior a 2,25 pontos. 


\section{Agradecimentos}

À FAPEMIG, pelo apoio financeiro na realização e publicação desta pesquisa.

\section{Referências}

BARUSELLI, P.S.; SÁ FILHO, M.F.; MARTINS C.M. et al. Superovulation and embryo transfer in Bos indicus cattle. Theriogenology, v.65, p.77-88, 2006.

BRITT, J.H. Enhanced reproduction and its economic implications. Journal of Dairy Science, v.68, p.1585-1592, 1985.

CARDOSO, B.L.; PESCARA, J.B.; VASCONCELOS, J.L.M. Protocolos de inseminação artificial em tempo fixo para vacas mestiças leiteiras. Acta Scientiae Veterinariae, v.34, (suppl. 1), p.428, 2006.

CHEBEL, R.C.; SANTOS, J.E.P.; REYNOLDS, J.P. et al. Factors affecting conception rate after artificial insemination and pregnancy loss in lactating dairy cows. Animal Reproduction Science, v.84, p.239-255, 2004.

DEMÉTRIO, D.G.B.; RODRIGUES, C.A.; SANTOS, R.M. et al. Factors affecting conception rates following artificial insemination or embryo transfer in lactating Holstein cows. Journal of Dairy Science, v.90, p.5073-5082, 2007.

DIAS, C.C.; WECHSLER, F.S.; DAY, M.L.; VASCONCELOS J.L.M. Progesterone concentrations, exogenous equine chorionic gonadotropin, and timing of prostaglandin F2a treatment affect fertility in postpuberal Nelore heifers. Theriogenology, v.72, p.378-385, 2009.

ESSLEMONT, R.J. Relationship between herd calving to conception interval and culling rate for failure to conceive. Veterinary Record, v.133, p.163-164, 1993.

FOLMAN, Y.; KAIM M.; HERZ, Z. et al. Comparison of methods for the synchronization of estrous cycles in dairy cows. 2. Effects of progesterone and parity on conception. Journal of Dairy Science, v.73, p.2817-2825, 1990.

FONSECA, F.A.; BRITT, J.H.; MCDANIEL, B.T.J. et al. Reproductive traits of Holsteins and Jerseys. Effects of age, milk yield, and clinical abnormalities on involution of cervix and uterus, ovulation, estrous cycles, detection of estrus, conception rate, and days open. Journal of Dairy Science, v.66, p.1128-1147, 1983.

FORTUNE, J.E. Ovarian follicular growth and development in mammals. Biology of Reproduction, v.50, p.225-232, 1994.

GALVÃO, K.N.; SANTOS, J.E.P.; JUCHEM, S.O. et al. Effect of addition of a progesterone intravaginal insert to a timed insemination protocol using estradiol cypionate on ovulation rate, pregnancy rate, and late embryonic loss in lactating dairy cows. Journal of Animal Science, v.82, p.3508-3517, 2004.

GROHN, Y.T.; RAJALA-SCHULTZ, P.J. Epidemiology of reproductive performance in dairy cows. Animal Reproduction Science, v.60-61, p.6505-6514, 2000.

HANSEN, P.J.; ARECHIGA, C.F. Strategies for managing reproduction in heat-stressed dairy cow. Journal of Animal Science, v.77, suppl. 2, p.36-50, 1999.
LOPEZ-GATIUS, F.; LOPEZ BEJAR, M.; FENECH, M. et al. Ovulation failure and double ovulation in dairy cattle: risks factors and effects. Theriogenology, v.63, p.1298-1307, 2005.

MARTEL, C.A. Fertility after timed AI insemination in response to a controlled internal drug release (CIDR) insert in lactating dairy cows. 2008. 48f. Thesis (MS Science) Kansas State University, Manhattan, Kansas, USA.

NEVES, J.P.; MIRANDA, K.L.; TORTORELLA, R.D. Progresso científico em reprodução na primeira década do século XXI. Revista Brasileira de Zootecnia, v.39, p.414-421, 2010 (supl. especial).

PERRY, G.A.; SMITH M.F.; GEARY, T.W. Ability of intravaginal progesterone inserts and melengestrol acetate to induce estrous cycles in postpartum beef cows. Journal of Animal Science, v.82, p.695-704, 2004.

PURSLEY, J.R.; WILTBANK, M.C.; STEVENSON, J.S. et al. Pregnancy rates per artificial insemination for cows and heifers inseminated at a synchronized ovulation or synchronized estrus. Journal of Dairy Science, v.80, p.295-300, 1997.

RIVERA, G.M.; GONI, C.G.; CHAVES, M.A. Ovarian follicular wave synchronization and induction of ovulation in postpartum beef cows. Theriogenology, v.49, p.1365-1376, 1998.

RUAS J.R.M.; MARCATTI NETO A.; AMARAL, R. et al. Programa de bovinos da EPAMIG - pesquisa com animais F1: projetos e resultados preliminares. In: ENCONTRO DE PRODUTORES DE GADO LEITEIRO F1, 4., 2002, Belo Horizonte. Anais... Belo Horizonte: UFMG, EV, 2002. p.60-68.

SANTOS, J.E.P.; RUTIGLIANO, H.M.; SÁ FILHO, M.F. Risk Factors for resumption of postpartum estrous cycles and embryonic survival in lactating dairy cows. Animal Reproduction Science, v.110, p.207-221, 2009.

SANTOS, R.M.; VASCONCELOS, J.L.M. Eficácia da dose reduzida de gonadorelina e diferentes prostaglandinas no protocolo ovsynch em vacas holandesas. Arquivo Brasileiro de Medicina Veterinária e Zootecnia, v.60, p.1323-1328, 2008.

SOUZA, A.H.; VIECHNIESKI, S.; LIMA, F.A. et al. Effects of equine chorionic gonadotropin and type of ovulatory stimulus in a timed-AI protocol on reproductive responses in dairy cows. Theriogenology, v.72, p.10-21, 2009.

STEVENSON, J.S.; CALL, E.P. Reproductive disorders in the periparturient dairy cow. Journal of Dairy Science, v.71, p.2572-2583, 1988.

TENHAGEN, B.A.; VOGEL C.; DRILLICH M. et al. Influence of stage of lactation and milk production on conception rates after timed artificial insemination following Ovsynch. Theriogenology, v.60, p.1527-1537, 2003.

VASCONCELOS, J.L.M.; GARCIA, P.H.M. Detecção de falha de ovulação permite re-sincronização rápida e aumento de vacas gestantes em 28 dias. In: REUNIÃO ANUAL DA SOCIEDADE BRASILEIRA DE TRANSFERÊNCIA DE EMBRIÕES, 20., 2006, Araxá. Anais... Porto Alegre: Acta Scientiae Veterinariae, 2006, v.34, (supl.1), p.292.

VASCONCELOS, J.L.M.; SILCOX, R.W.; ROSA, G.L.M. et al. Synchronization rate, size of the ovulatory follicle, and pregnancy rate after synchronization of ovulation beginning on different days of the estrous cycle in lactating dairy cows. Theriogenology, v.52, p.1067-1078, 1999. 